Pacific Journal of Mathematics

NUMERICAL INVARIANTS OF HOMOTOPIES INTO SPHERES 


\title{
NUMERICAL INVARIANTS OF HOMOTOPIES INTO SPHERES
}

\author{
JERROLd Siegel AND Frank Williams
}

\begin{abstract}
A classical problem in the calculus of variations may be expressed: Given two points in the same component of a Riemannian manifold $M$, what is the length of the shortest path connecting them? In this paper we discuss parametric analogs to this problem. The width of a homotopy is the supremum of the lengths of the paths traced by the points of $X$. Work of Allan Calder and the first author on the topology of Stone-Cech compactifications led to the study of the question: Given a space $X$ and two homotopic maps $f, g: X \rightarrow M$, what is the width of the shortest homotopy between them?

In this paper we obtain bounds $b_{q}(M)$ that depend only on the dimension $q$ of $X$ for the answer to this question in case $M$ is a sphere or a projective space (with the standard metric). We also introduce a related sequence of invariants $B_{q}(M)$ and compute these numbers for spheres and projective spaces. Of particular interest is the fact that $b_{2 n-2}\left(S^{n}\right)$ detects elements of Hopf invariant one while $B_{2 n-2}\left(S^{n}\right)$ does not.
\end{abstract}

One may regard this question as a parametric variational problem with parameters in the space $X$, or, equivalently, as a brachistochrone problem in the appropriately metrized function space $M^{X}$.

Of course in general the answer to the parametric problem may be infinity: If $X=\mathbf{R}$ and $M=S^{1}$, the complex numbers of unit norm, then the exponential map $\exp (t)=e^{2 \pi i t}$ is homotopic to the constant map, but not by a homotopy of finite width. However it was proved in [2] that $S^{1}$ is the exceptional case: If $X$ is a finite-dimensional normal space and $M$ is a compact Riemannian manifold with $\pi_{1}(M)$ finite, then any two homotopic maps $f$ and $g$ are connected by a homotopy of finite width.

Of more interest is the fact that these widths are bounded with respect to the dimension of $X$. In the classical problem above, if $M$ is compact then there is a bound $b_{0}(M)$ such that any two points in the same component are connected by a path of length $\leq b_{0}(M)$. (Simply take $b_{0}(M)$ to be the greatest of the diameters of the components of $M$ under the Riemannian distance.) The main theorem of [3] extended this result to homotopies: If $q \geq 0$ is an integer and $\pi_{1}(M)$ is finite, then there exists $a$ bound $\tilde{b}_{q}(M)$ such that any two homotopic maps $f, g: X \rightarrow M, \operatorname{dim}(M) \leq q$, are homotopic via a homotopy of width $\leq \tilde{b}_{q}(M)$. 
Consequently there is a sequence of invariants of $M$ defined by letting $b_{q}(M)=$ infimum of such $\tilde{b}_{q}(M)$. (Clearly this $b_{0}(M)$ coincides with the one defined above.) It was also shown in [3] that if $\operatorname{dim}(M)>0$ then $\lim _{q \rightarrow \infty} b_{q}(M)=\infty$, so that these invariants are non-trivial.

As a first step toward understanding these numbers, we began to calculate the $b_{q}\left(S^{n}\right)$, where $S^{n}$ is the standard unit sphere, $n \geq 2$. By a simplicial approximation argument one sees that $b_{q}\left(S^{n}\right)=\pi$ for $0 \leq q<n$, and some elementary algebraic topology shows that $b_{n}\left(S^{n}\right) \geq 2 \pi$, [3]. In [4] we computed all of the $b_{q}\left(S^{n}\right)$ with one exception (see Theorem 1.2 below). These numbers follow a somewhat regular pattern with exceptions when $q=n-1$ and when $q=2 n-2$ and $n=2,4$, or 8 .

In the present paper we obtain a better understanding of these invariants by introducing a new and independently interesting sequence of numbers $B_{q}(M)$ that do follow a regular pattern. $B_{q}(M)$ is the infimum of the numbers $\tilde{B}_{q}(M)$ such that any $H: X \times I \rightarrow M$, $\operatorname{dim}(X) \leq q$, can be deformed keeping the endmaps fixed into one of width less than $\tilde{B}_{q}(M)$. The proof in [3] of the finiteness of $b_{q}(M)$ actually shows that under the same hypotheses the numbers $B_{q}(M)$ are finite. (Variants of $B_{q}(M)$ have also been considered by $\mathrm{M}$. Gromov in [5].)

Clearly $b_{q}(M) \leq B_{q}(M)$. One would expect from the definitions that the inequality would usually be strict, but for spheres that turns out to be more the exception than the rule. In this paper we compute the $B_{q}\left(S^{n}\right)$, $q \geq 0, n \geq 2$, then use the results of this calculation to obtain the missing value, $b_{14}\left(S^{8}\right)$, via the solution of a differential equation in the Cayley bundle $S^{15} \rightarrow S^{8}$. Comparison of these results with those of [4] yields:

0.1. THEOREM. If $n \geq 2$, then $b_{q}\left(S^{n}\right)=B_{q}\left(S^{n}\right)$ unless $q=n-1$ or $n=2,4$, or 8 and $q=2 n-2$, in which cases $b_{q}\left(S^{n}\right)=B_{q-1}\left(S^{n}\right)<B_{q}\left(S^{n}\right)$.

Since the numbers $B_{q}\left(S^{n}\right)$ are easy to compute and describe (see Table I below), it would be desirable to have a direct proof of this theorem and to understand the relationship between $b_{q}(M)$ and $B_{q}(M)$ in general. We hope to deal with this question in the future. For the present, we do observe that $b_{2 n-2}\left(S^{n}\right)$ is sensitive to the presence of elements of Hopf invariant one in $\pi_{2 n-1}\left(S^{n-1}\right)$ and that $b_{q}\left(S^{n}\right)=B_{q}\left(S^{n}\right)$ except when the Hurewicz morphism $\pi_{q}\left(\Omega S^{n}\right) \rightarrow H_{q}\left(\Omega S^{n}\right)$ is a non-zero epimorphism. We conjecture:

0.2. Conjecture. Let $M$ be a compact Riemannian manifold with $\pi_{1}(M)$ finite. Then $b_{q}(M)$ is equal to either $B_{q}(M)$ or $B_{q-1}(M)$. It is equal to the 
former except when the Hurewicz morphism $\pi_{q}(\Omega M) \rightarrow H_{q}(\Omega M)$ is a nonzero epimorphism. In these cases $b_{q}(M)=B_{q-1}(M)$.

Further support for this conjecture comes through calculation of $B_{q}\left(P_{n}\right)$ and $b_{q}\left(P_{n}\right)$ for $P_{n}$ a real, complex, or quaternionic projective. We include in $\S 4$ a computation of $B_{q}\left(\mathbf{C} P_{n}\right)$ and $b_{q}\left(\mathbf{C} P_{n}\right)$ as a further example of our methods. The results appear in Table II.

\begin{tabular}{|l|c|c|}
\hline \multicolumn{1}{|c|}{$q$} & $b_{q}\left(S^{n}\right)$ & $B_{q}\left(S^{n}\right)$ \\
\hline $0 \leq q<n-1$ & $\pi$ & $\pi$ \\
\hline$q=n-1$ & $\pi$ & $2 \pi$ \\
\hline$n \leq q<2 n-2$ & $2 \pi$ & $2 \pi$ \\
\hline$q=2 n-2\left\{\begin{array}{l}n=2,4,8 \\
n \neq 2,4,8\end{array}\right.$ & $2 \pi$ & $3 \pi$ \\
\hline $2 n-2<q<3 n-3$ & $3 \pi$ & $3 \pi$ \\
\hline$(k-1)(n-1) \leq q<k(n-1), k \geq 4$ & $k \pi$ & $k \pi$ \\
\hline
\end{tabular}

\section{TABLE I}

Thus, for example, since $b_{22}\left(S^{7}\right)=4 \pi=B_{22}\left(S^{7}\right)$ any homotopy from a 22-dimensional complex $X$ to $S^{7}$ may be deformed (rel $X \times\{0,1\}$ ) into a homotopy of width artibrarily close to $4 \pi$. Furthermore, there exists a 22-complex $X$ and maps $f, g: X \rightarrow S^{7}$ such that $f$ and $g$ are homotopic but not by a homotopy of width less than $4 \pi$.

\begin{tabular}{|ccc|}
\hline$q$ & $b_{q}\left(\mathbf{C} P_{n}\right)$ & $B_{q}\left(\mathbf{C} P_{n}\right)$ \\
\hline 0 & $\pi / 2$ & $\pi / 2$ \\
\hline 1 & $\pi / 2$ & $\pi$ \\
\hline $2 \leq q \leq 2 n-1$ & $\pi$ & $\pi$ \\
\hline $2 n$ & $\pi$ & $3 \pi / 2$ \\
\hline $2(k-1) n<q<2 k n, k \geq 2$ & $k \pi$ & $k \pi$ \\
$2 k n, \quad k \geq 2$ & $(2 k+1) \pi / 2$ & $(2 k+1) / \pi / 2$ \\
\hline
\end{tabular}

\section{TABLE II}

1. Definitions and statement of results. Throughout this paper the domain space $X$ will be a finite-dimensional polyhedron. By standard "bridge" techniques [9] our results will also hold if $X$ is a finite-dimensional normal space. 
1.1. Definitions. Let $(M, d)$ be a metric space.

(a) The length $|\sigma|$ of a path $\sigma: I \rightarrow M$ is given by the formula

$$
|\sigma|=\sup \left\{\sum_{i=1}^{k} d\left(\sigma\left(t_{i}\right), \sigma\left(t_{i-1}\right)\right)\right\}
$$

taken over all partitions $0=t_{0}<t_{1}<\cdots<t_{k}=1$, for all $k$.

(b) The width $|H|$ of a homotopy $H: X \times I \rightarrow M$ is given by the formula $|H|=\sup \left\{\left|H_{x}\right|: x \in X\right\}$.

For maps $f, g: X \rightarrow M$ and a homotopy $H$ from $f$ to $g$, we consider the two extended real numbers:

(c) $W(H)=\inf \left\{\left|H^{\prime}\right|: H^{\prime} \sim H \operatorname{rel} X \times\{0,1\}\right\}$.

(d) $w(f, g)=\inf \left\{\left|H^{\prime}\right|: H_{0}^{\prime}=f, H_{1}^{\prime}=g\right\}$.

The subjects of this paper are the following sequences:

(e) $B_{q}(M)=\sup \{W(H): H: X \times I \rightarrow M, \operatorname{dim}(X) \leq q\}$.

(f) $b_{q}(M)=\sup \{w(f, g): f \sim g: X \rightarrow M, \operatorname{dim}(X) \leq q\}$.

Let $S^{n}$ denote the unit sphere in $R^{n+1}$. If $x, x^{\prime} \in S^{n}$, let $\operatorname{dist}\left(x, x^{\prime}\right)$ be the length of a minimum geodesic from $x$ to $x^{\prime}$. For the numbers $b_{q}\left(S^{n}\right)$, $n \geq 2$, we have the following result.

1.2. THEOREM [4]. If $(n, q)$ is a pair of integers such that $n \geq 2, q \geq 0$, and $(n, q)$ is not $(2,2),(4,6)$, or $(8,14)$, then

$$
b_{q}\left(S^{n}\right)= \begin{cases}\pi, & 0 \leq q \leq n-1 \\ 2 \pi, & n \leq q<2 n-2 \\ k \pi, & (k-1)(n-1) \leq q<k(n-1), k \geq 3 .\end{cases}
$$

Moreover, $b_{2}\left(S^{2}\right)=2 \pi=b_{6}\left(S^{4}\right)$ and $2 \pi \leq b_{14}\left(S^{8}\right) \leq 3 \pi$.

In $\S 3$ we shall show that:

1.3. THEOREM. The value of $b_{14}\left(S^{8}\right)$ is $2 \pi$.

The numbers $B_{q}\left(S^{n}\right)$ are relatively easy to compute. The following theorem will be proved in $\S 2$.

1.4. THEOREM. If $n, q$, and $k$ are integers such that $n \geq 2, k \geq 1$, and $(k-1)(n-1) \leq q<k(n-1)$, then $B_{q}\left(S^{n}\right)=k \pi$. 
We shall use the following kind of approximate fibration in the sequel:

1.5. Definition. Let $(M, d)$ be a metric space. A map $p: E \rightarrow M$ has the approximate covering homotopy property through dimension $k$, $\mathrm{XCHP}(k)$, provided that if $X$ is a normal space of dimension $\leq k$, and $G$ : $X \times I \rightarrow M$ and $g: X \rightarrow E$ are such that $p g(x)=G(x, 0)$, then for any $\varepsilon>0$ there exists $H: X \times I \rightarrow E$ such that $H(x, 0)=g(x)$ and $d(p H(x, t)$, $G(x, t))<\varepsilon$ for all $(x, t) \in X \times I$.

Let $E=\left\{\sigma: I \rightarrow S^{n}: \sigma\right.$ is piecewise $C^{\infty}$ with constant speed $\}$. We topologize $E$ as in $\left[8\right.$, p. 88]. For $0<c \leq \infty$ we define $E_{c}=\{\sigma \in E$ : $|\sigma|<c\}$ and let $p_{c}: E_{c} \rightarrow S^{n} \times S^{n}$ be given by $p_{c}(\sigma)=(\sigma(0), \sigma(1))$.

1.6. THEOREM [4, 1.4]. If $k \geq 0$ is an integer and $c>k \pi$ then $p_{c}$ : $E_{c} \rightarrow S^{n} \times S^{n}$ has the $\operatorname{XCHP}(k(n-1)-1)$.

2. Proof of 1.4. The following theorem will be used in this section to obtain the upper bounds for $B_{q}\left(S^{n}\right)$ and in $\S 3$ to compute $b_{14}\left(S^{8}\right)$.

2.1. TheOREM. Let $X$ be a complex of dimension less than $k(n-1)$, where $k \geq 1$ and $n \geq 2$. Suppose $H: X \times I \rightarrow S^{n}$ is a homotopy and $\varepsilon>0$. Then there exists $\bar{H}: X \times I \rightarrow S^{n}$ such that $\bar{H} \sim H(\operatorname{rel} X \times\{0,1\})$ and $|\bar{H}|<k \pi+\varepsilon$. Furthermore $\bar{H}$ may be chosen so that $\bar{H}_{x}$ is piecewise $C^{\infty}$ of constant speed for all $x \in X$.

Proof. Choose $\delta, \tau>0$ such that $\rho<\pi$ and $\delta+2 \rho<\varepsilon$. Define $G$ : $X \times I \rightarrow S^{n} \times S^{n}$ by $G(x, t)=(H(x, 0), H(x, t))$. Let $g: X \rightarrow E_{k \pi+\delta}$ be given by $g(x)=C[H(x, 0)]$, the constant path at $H(x, 0)$. By 1.6 there exists $\bar{G}: \quad X \times I \rightarrow E_{k \pi+\delta}$ such that $\bar{G}(x, 0)=g(x)$ and $\operatorname{dist}[(H(x, 0), H(x, t)), p \bar{G}(x, t)]<\rho$. Since $\rho<\pi$, any two points $y, y^{\prime}$ $\in S^{n}$ such that $\operatorname{dist}\left(y, y^{\prime}\right)<\rho$ may be joined by a unique minimal geodesic $m\left(y, y^{\prime}\right)$. Thus we may define $\hat{G}: X \times I \times I \rightarrow S^{n}$ by the formula

$$
\hat{G}(x, t, s)= \begin{cases}m(H(x, 0), \bar{G}(x, t)[0])[3 s], & 0 \leq s \leq \frac{1}{3}, \\ \bar{G}(x, t)[3 s-1], & \frac{1}{3} \leq s \leq \frac{2}{3} \\ m(\bar{G}(x, t)[1], H(x, t))[3 s-2], & \frac{2}{3} \leq s \leq 1 .\end{cases}
$$

Note that for each $(x, t) \in X \times I, \hat{G}_{(x, t)}$ is a piecewise $C^{\infty}$ path from $H(x, 0)$ to $H(x, t)$ of length less than $k \pi+\delta+2 \rho$, which is less than 
$k \pi+\varepsilon$. Let $\tilde{G}$ be $\hat{G}$ reparametrized so as to have constant speed in the $s$ parameter for each $(x, t) \in X \times I$.

We define $\bar{H}(x, s)$ to be $\tilde{G}(x, 1, s)$. A homotopy $K$ from $H$ to $\bar{H}$ is given by

$$
K(x, t, r)= \begin{cases}\tilde{G}(x, t, 1-2 r(1-t)), & 0 \leq r \leq \frac{1}{2} \\ \tilde{G}(x, t+(2 r-1)(1-t), t), & \frac{1}{2} \leq r \leq 1\end{cases}
$$

It is immediate that $K$ stays fixed on $X \times\{0,1\}$.

If we denote the dimension of $X$ by $q$, we see that a consequence of 2.1 is that $q<k(n-1)$ implies $B_{q}\left(S^{n}\right) \leq k \pi$.

The proof of 1.4 will be completed by showing that $B_{q}(M) \geq k \pi$ if $q \geq(k-1)(n-1)$. By 1.2 , unless $k=2$, or $n=2,4$, or 8 and $k=3$, we have $b_{q}\left(S^{n}\right) \geq k \pi$, whence $B_{q}\left(S^{n}\right) \geq k \pi$. Although we could dispose of the remaining numbers on a case-by-case basis, the following theorem gives us an easy proof of the desired inequality for all values of $q$ and $n$ at once, independent of any appeal to 1.2.

2.2. TheOREM. For all $n \geq 2, k \geq 1$, if $q \geq(k-1)(n-1)$, then $B_{q}\left(S^{n}\right) \geq k \pi$.

Proof. Let $\left(S^{n-1}\right)_{r}$ denote the $r$ th James reduced product space of $S^{n-1}$, [6], and let $E_{\lambda}(p, q)$ be the subspace of $E_{\lambda}$ consisting of paths from $p$ to $q$, for $p, q \in S^{n}$. Let $k$ be odd. In $[4,1.5]$ we noted that if $\lambda \leq k \pi$ then $E_{\lambda}(p,-p) \sim\left(S^{n-1}\right)_{r}$ for some $r<k-1$. Let $X=\left(S^{n-1}\right)_{k-1}$ and let $\eta$ : $\left(S^{n-1}\right)_{k-1} \rightarrow E(p,-p)$ be any map that is essential on homology in dimension $(k-1)(n-1)$. We consider $\eta$ as a homotopy between the constant maps of $X$ to $p$ and $-p$, respectively. If $\lambda=W(\eta) \leq k \pi$, then $\eta$ would factor up to homotopy through $E_{\lambda}(p,-p)$, contradicting the fact that $H_{(k-1)(n-1)}\left(E_{\lambda}(p,-p)\right)=0$. Thus $W(\eta)>k \pi$ and hence $B_{(k-1)(n-1)}\left(S^{n}\right) \geq k \pi$.

If $k$ is even we apply a similar argument to $E_{\lambda}(p, p)$.

3. The Cayley bundle. In this section we show that $b_{14}\left(S^{8}\right)=2 \pi$. Our proof involves a detailed study of the geometry of the Hopf-Cayley bundle. In fact our method also works for the Hopf complex and quaternionic bundles.

3.1. Notation. Let $K$ and $R$ denote the Cayley numbers and the real numbers. Let $S^{15}$ and $S^{8}$ be the unit spheres in $R^{16}=K \times K$ and 
$R^{9}=K \times R$. The Cayley bundle is given by the map $h: S^{15} \rightarrow S^{8}$, where

$$
h(c, d)=\left(2 c \bar{d},|c|^{2}-|d|^{2}\right) .
$$

We use juxtaposition to denote Cayley multiplication and "bar" to denote conjugation of Cayley numbers. We shall also use the vector dot product $c \cdot d$.

3.2. TheOREM. Suppose $F: X \times I \rightarrow S^{8}$ is a piecewise $C^{\infty}$ homotopy. Let $g: X \rightarrow S^{15}$ be such that $h g=F_{0}$. There exists $G: X \times I \rightarrow S^{15}$ such that $G_{0}=g, h G=F$; and for each $x, G_{x}$ is piecewise $C^{\infty}$ and $\left|G_{x}\right|=\frac{1}{2}\left|F_{x}\right|$ on common points of smoothness. Thus

$$
|G|=\frac{1}{2}|F| \text {. }
$$

Proof. Our proof amounts to showing that $h$ is a Riemannian submersion, [10]. This would be easy if $h$ were a principal bundle. The difficulty arises from the non-associativity of the Cayley numbers. We represent points of $S^{15}$ as $(u \cos \theta, v \sin \theta), u, v \in S^{7}$. The points of $S^{8}$ are represented as $(w \sin \Phi, \cos \Phi), w \in S^{7}$. With respect to this representation $h$ becomes

$$
h(u \cos \theta, v \sin \theta)=(u \bar{v} \sin 2 \theta, \cos 2 \theta) .
$$

For each point $x \in X$ we may write

$$
F(x, t)=(u(x, t) \sin [\psi(x, t)], \cos [\psi(x, t)]) .
$$

Locally

$$
g(x)=\left(u(x, 0) v(x, 0) \cos \left[\frac{1}{2} \psi(x, 0)\right], v(x, 0) \sin \left[\frac{1}{2} \psi(x, 0)\right]\right) .
$$

Moreover, any path covering $F$ is locally of the form

$$
G(x, t)=\left(u v \cos \left(\frac{1}{2} \psi\right), v \sin \left(\frac{1}{2} \psi\right)\right)
$$

where $v(x, t)$ is a path in $S^{7}$ starting at $v(x, 0)$. Thus to find a $g(x, t)$ that satisfies the requirements of 3.2 amounts to finding a suitable $v(x, t)$ such that

$$
|\dot{G}(x, t)|=\frac{1}{2}|\dot{F}(x, t)|
$$

Firstly $\dot{F}=(u \dot{\psi} \cos (\psi)+\dot{u} \sin (\psi),-\dot{\psi} \sin (\psi))$. Using the fact that $u \cdot \dot{u}=0($ since $|u|=1)$, we have

$$
|\dot{F}|^{2}=\frac{1}{4}(\dot{\psi})^{2}+|\dot{u}|^{2} \sin ^{2} \psi
$$


Similarly,

$$
|\dot{G}|^{2}=\frac{1}{4}(\dot{\psi})^{2}+|\dot{u}|^{2} \cos ^{2}(\psi / 2)+2(\dot{u} v) \cdot(u \dot{v}) \cos ^{2}(\psi / 2)+|\dot{v}|^{2} .
$$

By combining these last two relations, we see that 3.3 is equivalent to

$$
|\dot{u}|^{2} \cos (\psi / 2)+2(\dot{u} v) \cdot(u \dot{v}) \cos ^{2}(\psi / 2)+|\dot{v}|=\frac{1}{4} \sin ^{2}(\psi) .
$$

Using the identity $\cos ^{2}(\psi / 2)-\frac{1}{2} \sin ^{2}(\psi)=\cos ^{4}(\psi / 2)$, we reduce 3.4 to $\left|u \dot{v}+\dot{u} v \cos ^{2}(\psi / 2)\right|^{2}=0$. Hence it suffices to find $v$ such that

$$
u \dot{v}+\cos ^{2}(\psi / 2) \dot{u} v=0 .
$$

Since multiplication by $u$ is invertible as a linear operator, 3.5 may be considered to be a differential equation of the form $\dot{v}+A v=0$, where $A$ is a continuous family of linear operators. It is a standard result that such a system with continuous initial conditions $v(x, 0)$ has a solution $v(x, t)$ that is continuous in $x$. (See for example [1; Theorem 3.8].)

Finally we observe that dotting 3.5 with $u v$ yields $v \cdot \dot{v}=0$ so $v(x, t) \in S^{7}$. For this last observation we use the relation $(a \cdot b)|c|^{2}=$ $a c \cdot b c$.

3.6. Notation. In order to define our homotopies it will be convenient to have a radial coordinate for our standard 14-cell. To this end let $S^{13}$ be a 13-sphere and $D^{14}$ be the cone on $S^{13}, D^{14}=\left(I \times S^{13}\right) /\left(\{0\} \times S^{13}\right)$. Write $x=s \wedge y$ for points of $D^{14}$, where $(s, y) \in I \times S^{13}$.

3.7. TheOREM. Let $F: D^{14} \times I \rightarrow S^{8}$ be such that $F \mid S^{13} \times I$ is piecewise $C^{\infty}$ and of width less than $2 \pi$. Let $\varepsilon>0$. Then there exists $\bar{F}$ : $D^{14} \times I \rightarrow S^{8}$ such that
(a) $|\bar{F}|<2 \pi$,
(b) $\bar{F}=F$ on $S^{13} \times I$,
(c) $\operatorname{dist}(\bar{F}(x, i), F(x, i))<\varepsilon, x \in D^{14}, i=0,1$.

Proof. Since $D^{14}$ is contractible, $F \mid D^{14} \times\{0\}$ lifts to $G: D^{14} \times\{0\} \rightarrow$ $S^{15}$. By 3.2 we may extend $G$ to a lift of $F \mid\left(\left(S^{13} \times I\right) \cup\left(D^{14} \times\{0\}\right)\right)$ such that $\left|G_{y}\right|=\frac{1}{2}\left|F_{y}\right|<\pi$ for every $y \in S^{13}$. Now apply the covering homotopy extension property to lift $F$ to all of $D^{14} \times I$.

If $y \in S^{13}$ then $G(y, 0) \neq-G(y, 1)$. Thus by dimensionality we may deform $G$ to $\tilde{G}$ so that $G=\tilde{G}$ on $S^{13} \times I$, $\operatorname{dist}(G(x, i), \tilde{G}(x, i))<\varepsilon / 4$ for all $(x, i) \in D^{14} \times\{0,1\}$ and $\tilde{G}(x, 0) \neq-\tilde{G}(x, 1)$ for all $x$.

Let $0<s_{0}<1$ be small enough so that
(a) $\operatorname{dist}(\tilde{G}(s \wedge y, i), \tilde{G}(1 \wedge y, i))<\varepsilon / 4 ; y \in S^{13}, s_{0} \leq s \leq 1, i=0,1$, 
and

(b) $\operatorname{dist}\left(\tilde{G}(s \wedge y, i), \tilde{G}\left(s / s_{0} \wedge y, i\right)\right)<\varepsilon / 4 ; y \in S^{13}, 0 \leq s \leq s_{0}, i=$ 0,1 .

Define

$$
\hat{G}(s \wedge y, i)= \begin{cases}\tilde{G}(1 \wedge y, i), & s_{0} \leq s \leq 1, \\ \tilde{G}\left(s / s_{0} \wedge y, i\right), & 0 \leq s \leq s_{0} .\end{cases}
$$

For $s \wedge y \in D^{14}$ and $t \in I$, let

$$
\begin{aligned}
& \bar{G}(s \wedge y, t) \\
& \quad= \begin{cases}m(\hat{G}(s \wedge y, 0), \hat{G}(s \wedge y, 1))[t], & 0 \leq s \leq s_{0}, 0 \leq t \leq 1, \\
m\left(G(1 \wedge y, 0), G\left(1 \wedge y, \frac{1-s}{1-s_{0}}\right)\right)\left[\frac{1-s_{0}}{1-s} t\right], & s_{0} \leq s \leq 1,0 \leq t \leq \frac{1-s}{1-s_{0}}, \\
G(1 \wedge y, t), & s_{0} \leq s \leq 1, \frac{1-s}{1-s_{0}} \leq t \leq 1 .\end{cases}
\end{aligned}
$$

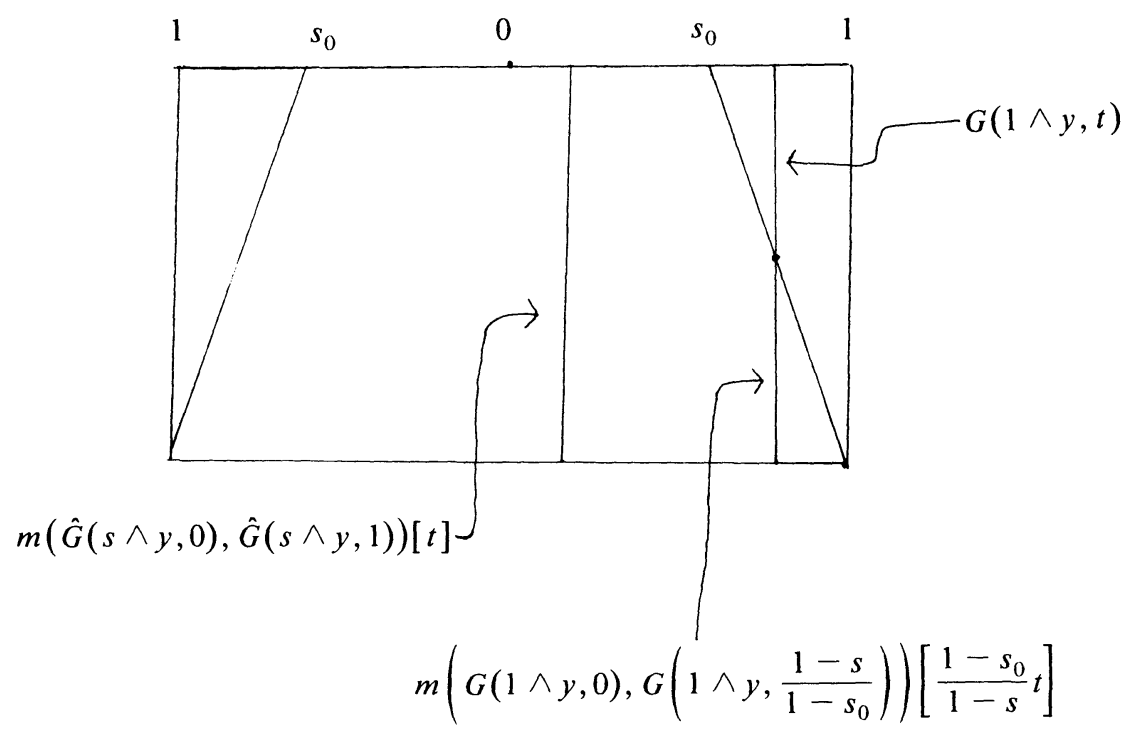

Figure 1

Note that $\bar{G}=G$ on $S^{13} \times I$ and $\operatorname{dist}(\bar{G}(x, i), G(x, i))<\varepsilon / 2$ for $x \in D^{14}, i=0,1$. Moreover if $0 \leq s \leq s_{0}$, then

$$
\left|\bar{G}_{s \wedge y}\right|=\operatorname{dist}(\hat{G}(s \wedge y, 0), \hat{G}(s \wedge y, 1))<\pi .
$$


Finally, if $s_{0} \leq s \leq 1$, then

$$
\begin{aligned}
\left|\bar{G}_{s \wedge y}\right| & =\left|m\left(G(1 \wedge y, 0), G\left(1 \wedge y, \frac{1-s}{1-s_{0}}\right)\right)\right|+\left|G_{1 \wedge y}\left[\frac{1-s}{1-s_{0}}, 1\right]\right| \\
& \leq\left|G_{1 \wedge y}\left[0, \frac{1-s}{1-s_{0}}\right]\right|+\left|G_{1 \wedge y}\left[\frac{1-s}{1-s_{0}}, 1\right]\right|=\left|G_{1 \wedge y}\right|<\pi,
\end{aligned}
$$

where $G_{1 \wedge y}\left[s_{1}, s_{2}\right]$ denotes the restriction of the path $G_{1 \wedge y}$ to the subinterval $\left[s_{1}, s_{2}\right]$.

Set $\bar{F}=h \bar{G}$. Since the dilatation of $h$ is $2, \quad|\bar{F}|<2 \pi$ and $\operatorname{dist}(F(x, i), \bar{F}(x, i))<\varepsilon, i=0,1$. By definition, $F=\bar{F}$ on $S^{13} \times I$.

3.8. TheOREM. Let $X$ be a 14-dimensional complex, $f, f^{\prime}: X \rightarrow S^{8}$ be homotopic maps, and $\varepsilon>0$. Then $f$ and $f^{\prime}$ are homotopic by a homotopy of width less than $2 \pi+\varepsilon$. Hence $b_{14}\left(S^{8}\right) \leq 2 \pi$.

Proof. Let $F: X \times I \rightarrow S^{8}$ be a homotopy of $f$ to $f^{\prime}$ and let $0<\varepsilon_{1}<\varepsilon / 8$. If we let $X^{(13)}$ denote the 13-skeleton of $X$, we may apply 3.1 to obtain $\bar{F}$ : $X^{(13)} \times I \rightarrow S^{8}$ such that $\bar{F}$ is piecewise $C^{\infty}$ of constant speed, of width less than $2 \pi+\varepsilon_{1}$, and homotopic to $F\left(\right.$ rel $\left.X^{(13)} \times\{0,1\}\right)$. We now use the homotopy extension property to extend $\bar{F}$ to a homotopy from $f$ to $f^{\prime}$.

We define $G: X^{(13)} \times I \rightarrow S^{8}$ by

$$
G(x, t)=\bar{F}\left(x, \frac{2 \pi-\varepsilon_{1}}{2 \pi+\varepsilon_{1}} t\right) .
$$

Observe that $|G| \leq 2 \pi-\varepsilon_{1}<2 \pi$ and $\operatorname{dist}\left(G(x, 1), f^{\prime}(x)\right)<\varepsilon / 4$. By a patching process similar to that used in the proof of 3.7 we extend $G$ to $K \times I$ such that $G(x, 0)=f(x)$ and $\operatorname{dist}\left(g(x, 1), f^{\prime}(x)\right)<\varepsilon / 4$ for all $x \in X$.

We next apply 3.7 to $G$ on the individual 14-cells of $X$, using $\varepsilon / 4$ in place of $\varepsilon$. Since 3.7 leaves $G$ unchanged on the boundary of each 14-cell, the resulting homotopy $\bar{G}: X \times I \rightarrow S^{8}$ is such that $\bar{G}=G$ on $X^{(13)} \times I$, $|\bar{G}| \leq 2 \pi$, and $\operatorname{dist}(\bar{G}(x, i))<\varepsilon / 4$, for all $x \in X$ and $i=0,1$.

Now $\operatorname{dist}(\bar{G}(x, 0), f(x))<\varepsilon / 4$ and

$$
\begin{aligned}
\operatorname{dist}\left(\bar{G}(x, 1), f^{\prime}(x)\right) & \leq \operatorname{dist}(\bar{G}(x, 1), G(x, 1))+\operatorname{dist}\left(G(x, 1), f^{\prime}(x)\right) \\
& <\varepsilon / 4+\varepsilon / 4=\varepsilon / 2 .
\end{aligned}
$$

If we compose $\bar{G}$ with the minimal geodesics on each end from $f(x)$ to $\bar{G}(x, 0)$ and from $\bar{G}(x, 1)$ to $g(x)$, as in the proof of 3.1 , we obtain a homotopy $\hat{G}$ from $f$ to $f^{\prime}$ such that $|G| \leq 2 \pi+3 \varepsilon / 4<2 \pi+\varepsilon$.

Since 1.2 tells us that $b_{14}\left(S^{8}\right) \geq 2 \pi$, the proof of 1.3 is accomplished. 
4. Projective spaces. In this section we shall compute the invariants for complex projective spaces (with the metric induced from the Hopf map $S^{2 n+1} \rightarrow \mathbf{C} P_{n}$ ). We shall proceed as for spheres, in that we shall calculate upper and lower bounds for $B_{q}\left(\mathbf{C} P_{n}\right)$ and lower bounds for $b_{q}\left(\mathbf{C} P_{n}\right)$. When these do not coincide we shall use special arguments.

The geodesic structure of $\mathbf{C} P_{n}$ is well known, see e.g. [7]. Let $E$ be the space of piecewise $C^{\infty}$ paths of constant speed and $E \rightarrow \mathbf{C} P_{n} \times \mathbf{C} P_{n}$ be the projection, as in the paragraph preceding 1.6. Since $\Omega \mathrm{C} P_{n} \sim S^{1} \times \Omega S^{2 n+1}$, it follows that for $p, q \in \mathbf{C} P_{n}, E_{\lambda}(p, q)$ is homotopic to a skeleton $K^{(m)}$ of $K=S^{1} \times\left(S^{2 n}\right)_{\infty}$. Indeed, if $\lambda \leq k \pi$, then $E_{\lambda}(p, p) \sim K^{(m)}$ for some $m \leq 2(k-1) n$. Furthermore, regarding $p$ as a complex line in $\mathbf{C}^{n}$, if $p^{\prime}$ is an orthogonal line and $\lambda \leq(2 k+1) \pi / 2$, then $E_{\lambda}\left(p, p^{\prime}\right) \sim K^{(m)}$ for some $m \leq 2 k n-1$. Thus, as in 2.2 , we have:
4.1. THEOREM. (a) If $q \geq 2(k-1) n+1$, then $B_{q}\left(\mathbf{C} P_{n}\right) \geq k \pi$.
(b) If $q \geq 2 k n$, then $B_{q}\left(\mathbf{C} P_{n}\right) \geq(2 k+1) \pi / 2$.

Moving to upper bounds on $B_{q}\left(\mathbf{C} P_{n}\right)$, we have from Morse theory:

4.2. (a) If $\lambda>k \pi$, then for all $p, p^{\prime}, E_{\lambda}\left(p, p^{\prime}\right) \sim K^{(m)}$ for some $m \geq 2 k n-1$.

(b) If $\lambda>(2 k+1) \pi / 2$, then for all $p, p^{\prime}, E_{\lambda}\left(p, p^{\prime}\right) \sim K^{(m)}$ for some $m \geq 2 k n$.

Thus in these cases, as in $[4,1.4], E_{\lambda} \rightarrow \mathbf{C} P_{n} \times \mathbf{C} P_{n}$ has the $\operatorname{XCHP}(m)$ for the appropriate $m$. Hence:
4.3. TheOREM. (a) If $q \leq 2 k n-1$, then $B_{q}\left(\mathbf{C} P_{n}\right) \leq k \pi$.
(b) If $q \leq 2 k n$, then $B_{q}\left(\mathbf{C} P_{n}\right) \leq(2 k+1) \pi / 2$.

Combining 4.1 and 4.3 , we obtain the values for $B_{q}\left(\mathbf{C} P_{n}\right)$ listed in Table II.

The lower bounds for $b_{q}\left(\mathbf{C} P_{n}\right)$ are somewhat more complicated. We content ourselves here by referring to [4, pp. 27-289], since the method of computation used there applies in our present situation. The results here are somewhat simpler since for all $n$, the Hurewicz morphism $\pi_{r}\left(\Omega \mathbf{C} P_{n}\right) \rightarrow$ $H_{r}\left(\Omega C P_{n}\right)$ is zero except when $r=1$ or $2 n$. We obtain:

4.4. THEOREM. If $q \neq 1$ or $2 n$, then $b_{q}\left(\mathbf{C} P_{n}\right)=B_{q}\left(\mathbf{C} P_{n}\right)$.

If $q=1$, the fact that $\pi_{1}\left(\mathbf{C} P_{n}\right)=0$ yields

$$
b_{1}\left(\mathbf{C} P_{n}\right)=\operatorname{diam}\left(\mathbf{C} P_{n}\right)=\pi / 2 \text {. }
$$


If $q=2 n$, we consider the bundle $S^{1} \rightarrow S^{2 n+1} \rightarrow \mathbf{C} P_{n}$. Since this bundle is principal and $S^{1}$ operates by isometries, the argument of [4, Theorem 2.3] shows that $b_{2 n}\left(\mathbf{C} P_{n}\right) \leq b_{2 n}\left(S^{2 n+1}\right)=\pi$. Hence:

\subsection{THEOREM. $b_{2 n}\left(\mathbf{C} P_{n}\right)=\pi$.}

We conclude by observing that the same method also yields the values of $b_{q}$ and $B_{q}$ for the real and quaternionic projective spaces. In all these examples, as in the case of spheres, we are able to compute the values of $B_{q}$ since the upper and lower bounds we obtain for $B_{q}$ coincide with each other. A goal for future work is a characterization of those spaces for which this phenomenon occurs and, if possible, to discover the relationship between $b_{q}, B_{q}$, and the topology of the space of loops on the manifold.

\section{REFERENCES}

[1] Brauer and Nohel, Qualitative Theory of Differential Equations, Benjamin 1969.

[2] A. Calder and J. Siegel, Homotopy and uniform homotopy, Trans. Amer. Math. Soc., 235 (1978), 245-270.

[3] _ On the width of homotopies, Topology 19 (1980), 209-220.

[4] A. Calder, J. Siegel and F. Williams, The width of homotopies into spheres, Topology, 21 (1982), 281-290.

[5] M. Gromov, Homotopical effects of dilatation, J. Differential Geom., 13 (1978), 303-310.

[6] I. M. James, Reduced product spaces, Ann. Math., 62 (2) (1955), 170-197.

[7] S. Kobayashi and K. Nomizu, Foundations of Differential Geometry, Vol. 2, Interscience, New York, 1969.

[8] J. Milnor, Morse Theory, Annals of Mathematics Studies Number 51, Princeton University, 1963.

[9] K. Morita, Cech cohomology and covering dimension for topological spaces, Adv. in Math., 15 (1975), 198-206.

[10] B. O'Neill, The fundamental equations of a submersion, Michigan Math. J., 13 (1966), 459-469.

Received December 7, 1982.

UNIVERSITY OF MISSOURI

ST. Louis, MO 63121

AND

New Mexico State University

LAS CRUCES, NM 88003 


\title{
PACIFIC JOURNAL OF MATHEMATICS EDITORS
}

\author{
DONALD BABBITT (Managing Editor) \\ University of California \\ Los Angeles, CA 90024 \\ Hugo Rossi \\ University of Utah \\ Salt Lake City, UT 84112 \\ C. C. MOORE and Arthur Ogus \\ University of California \\ Berkeley, CA 94720
}

\author{
J. Dugundir \\ Department of Mathematics \\ University of Southern California \\ Los Angeles, CA 90089-1113
}

R. FINN and H. SAMELSON

Stanford University

Stanford, CA 94305

ASSOCIATE EDITORS
R. ARENS
E. F. BECKENBACH
B. H. NeumanN
F. WOLF
K. YosHIDA (1906-1982)

\section{SUPPORTING INSTITUTIONS}

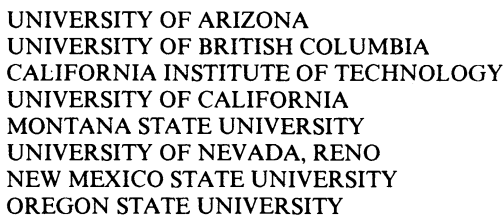

\author{
UNIVERSITY OF OREGON \\ UNIVERSITY OF SOUTHERN CALIFORNIA \\ STANFORD UNIVERSITY \\ UNIVERSITY OF HAWAII \\ UNIVERSITY OF TOKYO \\ UNIVERSITY OF UTAH \\ WASHINGTON STATE UNIVERSITY \\ UNIVERSITY OF WASHINGTON
}

The Supporting Institutions listed above contribute to the cost of publication of this Journal, but they are not owners or publishers and have no responsibility for its content or policies.

Mathematical papers intended for publication in the Pacific Journal of Mathematics should be in typed form or offset-reproduced (not dittoed), double spaced with large margins. Please do not use built up fractions in the text of the manuscript. However, you may use them in the displayed equations. Underline Greek letters in red, German in green, and script in blue. The first paragraph must be capable of being used separately as a synopsis of the entire paper. In particular it should contain no bibliographic references. Please propose a heading for the odd numbered pages of less than 35 characters. Manuscripts, in triplicate, may be sent to any one of the editors. Please classify according to the scheme of Math. Reviews, Index to Vol. 39. Supply name and address of author to whom proofs should be sent. All other communications should be addressed to the managing editor, or Elaine Barth, University of California, Los Angeles, California 90024.

There are page-charges associated with articles appearing in the Pacific Journal of Mathematics. These charges are expected to be paid by the author's University, Government Agency or Company. If the author or authors do not have access to such Institutional support these charges are waived. Single authors will receive 50 free reprints; joint authors will receive a total of 100 free reprints. Additional copies may be obtained at cost in multiples of 50 .

The Pacific Journal of Mathematics is issued monthly as of January 1966. Regular subscription rate: \$132.00 a year (6 Vol., 12 issues). Special rate: $\$ 66.00$ a year to individual members of supporting institutions.

Subscriptions, orders for numbers issued in the last three calendar years, and changes of address should be sent to Pacific Journal of Mathematics, P.O. Box 969, Carmel Valley, CA 93924, U.S.A. Old back numbers obtainable from Kraus Periodicals Co., Route 100, Millwood, NY 10546.

The Pacific Journal of Mathematics ISSN 0030-8730 is published monthly by the Pacific Journal of Mathematics at P.O. Box 969, Carmel Valley, CA 93924. Application to mail at Second-class postage rates is pending at Carmel Valley, California, and additional mailing offices. Postmaster: Send address changes to Pacific Journal of Mathematics, P. O. Box 969, Carmel Valley, CA 93924.

PUBLISHED BY PACIFIC JOURNAL OF MATHEMATICS, A NON-PROFIT CORPORATION

Copyright $(1984$ by Pacific Journal of Mathematics 


\section{Pacific Journal of Mathematics}

Vol. 110, No. $2 \quad$ October, 1984

Robert A. Bekes, The range of convolution operators $\ldots \ldots \ldots \ldots \ldots 257$

Dennis K. Burke and Sheldon Davis, Subsets of ${ }^{\omega} \omega$ and generalized metric

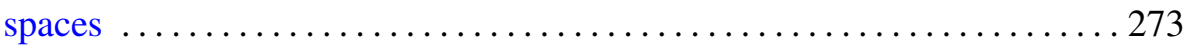

Giovanni Emmanuele, A remark on a paper: "Common fixed points of nonexpansive mappings by iteration" $\ldots \ldots \ldots \ldots \ldots \ldots \ldots \ldots \ldots 283$

I. Erdélyi and Sheng-Wang Wang, On strongly decomposable operators . . . 287

Gerhard Gierz, Injective Banach lattices with strong order units . . . . . . . 297

Maurizio Letizia, Quotients by complex conjugation of nonsingular quadrics and cubics in $\mathbf{P}_{\mathbf{C}}^{3}$ defined over $\mathbf{R} \ldots \ldots \ldots \ldots \ldots \ldots \ldots \ldots \ldots \ldots$

P. H. Maserick and Franciszek Hugon Szafraniec, Equivalent definitions

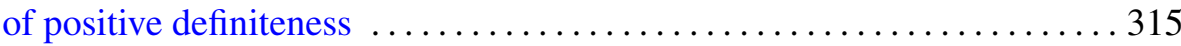

Costel Peligrad and S. Rubinstein, Maximal subalgebras of $C^{*}$-crossed

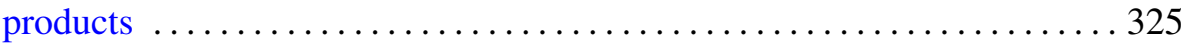

Derek W. Robinson and Sadayuki Yamamuro, Hereditary cones, order

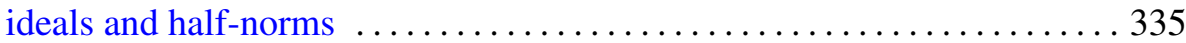

Derek W. Robinson and Sadayuki Yamamuro, The Jordan decomposition

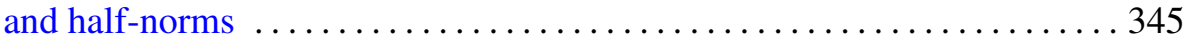

Richard Rochberg, Interpolation of Banach spaces and negatively curved

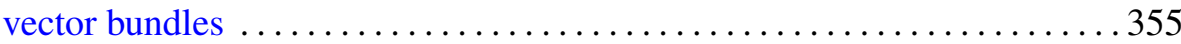

Dale Rolfsen, Rational surgery calculus: extension of Kirby's theorem 377

Walter Iaan Seaman, Helicoids of constant mean curvature and their Gauss maps

Diana Shelstad, Endoscopic groups and base change $\mathbf{C} / \mathbf{R}$

Jerrold Norman Siegel and Frank Williams, Numerical invariants of homotopies into spheres

Alladi Sitaram, Some remarks on measures on noncompact semisimple Lie groups

Teruhiko Soma, Atoroidal, irreducible 3-manifolds and 3-fold branched coverings of $S^{3}$

Jan de Vries, On the $G$-compactification of products

Hans Weber, Topological Boolean rings. Decomposition of finitely additive set functions 